Supporting information to:

\title{
Triple isotope fractionation exponents of elements measured by MC-ICP-MS - an example of Mg
}

Published in: Analytical Chemistry; DOI: 10.1021/acs.analchem.9b02699

Michael Tatzel ${ }^{1 *} \uparrow$, Jochen Vogl ${ }^{1}$, Martin Rosner ${ }^{2}$, Michael J. Henehan $^{3}$, Thomas Tütken ${ }^{4}$

${ }^{1}$ Bundesanstalt für Materialforschung und -prüfung (BAM), Richard-Willstätter Str. 11, 12489 Berlin, Germany

${ }^{2}$ IsoAnalysis UG, 10829 Berlin, Germany

${ }^{3}$ GFZ German Research Centre for Geosciences, 14473 Potsdam, Germany

${ }^{4}$ Institute of Geosciences, Applied and Analytical Palaeontology, University of Mainz, 55128 Mainz, Germany

* corresponding author. E-Mail: mtatzel@ucsc.edu

$\dagger$ Department of Earth and Planetary Sciences, University of California Santa Cruz, 1156 High Street, Santa Cruz, CA 95064, USA (present address)

This Supporting Information includes a supporting text detailing the uncertainty contributions to (apparent) $\theta$-values and their calculations (with equations $\mathrm{S} 1$ to S8) and a supporting text on the precision of apparent $S$-values (with fig. S1). Data shown in the main text is presented in form of data tables $\mathrm{S} 1$ to $\mathrm{S} 4$. The equation for converting $\delta$-values is provided in equation S9. Theoretical $\theta$-values for different mass-dependent isotope fractionation processes are shown in figure S2; a schematic mass-spectrum of $\mathrm{Mg}$ is shown in figure $\mathrm{S} 3$, the impact of interferences on $\Delta^{6}$-values in figure S4. 


\section{Supporting Text}

\section{Contribution to uncertainty}

For the standard uncertainty on the isotope ratio measurement, we used the standard error of an individual measurement (often termed internal standard error). We used a value of approx. $4 \times 10^{-5}$, which is significantly larger than typical standard errors on the order of $5 \times 10^{-6} . \kappa_{1}$ and $\kappa_{2}$ are calculated based on the isotopic difference between a repeatedly processed and measured reference sample and its published average $\delta$-value. We used the dolomite standard JDo-1 with published average $\delta^{25 / 24} \mathrm{Mg}_{\mathrm{DSM} 3}=-1.23 \%$ and $\delta^{26 / 24} \mathrm{Mg}_{\mathrm{DSM} 3}=-2.35 \%{ }^{1}$ that we ourselves measured as $-1.20 \% \mathrm{o} \pm$ $0.04 \%$ and $-2.32 \% \pm 0.05 \%$ ( $2 \mathrm{SD}, \mathrm{n}=14)$, respectively. Thus, we estimate $\kappa_{1}$ and $\kappa_{2}$ to $0.015 \%$, which is half of the difference between the measured and the reference value. We estimated the contribution of the mass spectrometric background $\left(\kappa_{3}\right)$ of $0.0136 \%$, based on the average blank-tosignal ratio of all isotopes within one representative measurement session. This estimate is a worstcase scenario that includes the procedural blank (approx. $7 \mathrm{ng} \mathrm{Mg}$ ) and a typical analyte intensity-toacid blank ratio $(>1000)$. We estimate the uncertainty contribution through heterogeneity of the bracketing material $\left(\kappa_{4}\right)$ to be $0.0069 \%$ and $0.01 \%$ for $\delta^{25 / 24} \mathrm{Mg}$ and $\delta^{26 / 24} \mathrm{Mg}$, based on homogeneity tests on the standards ERM-AE143, ERM-AE144, and ERM-AE1452. We estimated the impact of mass bias drift $\left(\kappa_{5}\right)$ from the drift between two bracketing standards, calculated as the total mass bias drift of a representative analytical session $(0.9 \%)$ divided by the number of standards analyzed $(\mathrm{n}=$ 103). This approach yields a drift contribution of $0.0043 \%$ for $\delta^{25 / 24} \mathrm{Mg}$ and $0.0087 \%$ for $\delta^{26 / 24} \mathrm{Mg}$. The offset in mass bias related to matrix changes $(\kappa 6)$ we estimate conservatively based on a maximum $10 \%$ mismatch in acid concentration that would cause an uncertainty of $0.0088 \% \delta^{25 / 24} \mathrm{Mg}$ and $0.0175 \% \delta^{26 / 24} \mathrm{Mg}$ (based on our own measurements). We estimate the uncertainty contribution from residual interferences $\left(\kappa_{7}\right)$ to be $0.001 \%$, corresponding to an interference-to-signal ratio of $10^{-6}$ (see section 2.3). 


\section{Calculation of uncertainties on the $\theta$-value}

When a $\theta$-value is based on two samples where both have been analyzed by standard-sample bracketing, the uncertainty on $\theta$ comprises uncertainty contributions from two $\delta^{\prime 25 / 24} \mathrm{Mg}$-values and two $\delta^{26 / 24} \mathrm{Mg}$-values (supporting equation $\mathrm{S} 1$ ).

$$
\begin{aligned}
u^{2}(\theta) & =\left(\frac{\partial \theta}{\partial \delta^{25 / 24} M g(A)_{s t d}} \cdot u\left(\delta^{25 / 24} M g(A)_{s t d}\right)\right)^{2}+\left(\frac{\partial \theta}{\partial \delta^{26 / 24} M g(A)_{s t d}} \cdot u\left(\delta^{26 / 24} M g(A)_{s t d}\right)\right)^{2} \\
& +\left(\frac{\partial \theta}{\partial \delta^{25 / 24} M g(B)_{s t d}} \cdot u\left(\delta^{25 / 24} M g(B)_{s t d}\right)\right)^{2}+\left(\frac{\partial \theta}{\partial \delta^{26 / 24} M g(B)_{s t d}} \cdot u\left(\delta^{26 / 24} M g(B)_{s t d}\right)\right)^{2} \\
& +2 \cdot\left(\frac{\partial \theta}{\partial \delta^{25 / 24} M g(A)_{s t d}} \cdot \frac{\partial \theta}{\partial \delta^{26 / 24} M g(A)_{s t d}} \cdot u\left(\delta^{25 / 24} M g(A)_{s t d}\right) \cdot u\left(\delta^{26 / 24} M g(A)_{s t d}\right) \cdot \mathrm{r}\right) \\
& +2 \cdot\left(\frac{\partial \theta}{\partial \delta^{25 / 24} M g(B)_{s t d}} \cdot \frac{\partial \theta}{\partial \delta^{26 / 24} M g(B)_{s t d}} \cdot u\left(\delta^{25 / 24} M g(B)_{s t d}\right) \cdot u\left(\delta^{26 / 24} M g(B)_{s t d}\right) \cdot \mathrm{r}\right)
\end{aligned}
$$

Where $\mathrm{r}$ is the empirical correlation coefficient. The partial derivatives used for the calculation of the uncertainty on $\theta$ are as follows (Supporting equations S2 to S5):

$$
\begin{aligned}
& \frac{\partial \theta}{\partial \delta^{\frac{25}{24}} M g(A)_{s t d}} \\
& =\frac{1}{\ln \left(\left(\delta^{\frac{26}{24}} M g(A)_{s t d}+1000\right)-\ln \left(\delta^{\frac{26}{24}} M g(B)_{s t d}+1000\right)\right)} \cdot \frac{1}{\left(\delta^{\frac{25}{24}} M g(A)_{s t d}+1000\right)} \\
& \frac{\partial \theta}{\partial \delta^{\frac{25}{24}} M g(B)_{s t d}} \\
& =\frac{1}{\ln \left(\left(\delta^{\frac{26}{24}} M g(A)_{s t d}+1000\right)-\ln \left(\delta^{\frac{26}{24}} M g(B)_{s t d}+1000\right)\right)} \cdot \frac{1}{\left(\delta^{\frac{25}{24}} M g(B)_{s t d}+1000\right)}
\end{aligned}
$$

$$
\begin{aligned}
& \frac{\partial \theta}{\partial \delta^{\frac{26}{24}} M g(A)_{s t d}} \\
& =-\frac{\left(\ln \left(\delta^{\frac{25}{24}} M g(A)_{s t d}+1000\right)-\ln \left(\delta^{\frac{25}{24}} M g(B)_{s t d}+1000\right)\right)}{\left(\ln \left(\delta^{\frac{26}{24}} M g(A)_{s t d}+1000\right)-\ln \left(\delta^{\frac{26}{24}} M g(B)_{s t d}+1000\right)\right)^{2}} \cdot \frac{1}{\left(\delta^{\frac{26}{24}} M g(A)_{s t d}+1000\right)}
\end{aligned}
$$




$$
\begin{aligned}
& \frac{\partial \theta}{\partial \delta^{\frac{26}{24}} M g(B)_{s t d}} \\
& =\frac{\left(\ln \left(\delta^{\frac{25}{24}} M g(A)_{s t d}+1000\right)-\ln \left(\delta^{\frac{25}{24}} M g(B)_{s t d}+1000\right)\right)}{\left(\ln \left(\delta^{\frac{26}{24}} M g(A)_{s t d}+1000\right)-\ln \left(\delta^{\frac{26}{24}} M g(B)_{s t d}+1000\right)\right)^{2}} \cdot \frac{1}{\left(\delta^{\frac{26}{24}} M g(B)_{s t d}+1000\right)}
\end{aligned}
$$

Using sample-sample bracketing, the uncertainty on the $\theta$-value will be reduced, because only two $\delta$ value measurements contribute to the uncertainty budget of $\theta$ (equation S6).

$$
\begin{aligned}
u^{2}(\theta) & =\left(\frac{\partial \theta}{\partial \delta^{25 / 24} M g(A)_{B}} \cdot u\left(\delta^{25 / 24} M g(A)_{B}\right)\right)^{2}+\left(\frac{\partial \theta}{\partial \delta^{26 / 24} M g(A)_{B}} \cdot u\left(\delta^{26 / 24} M g(A)_{B}\right)\right)^{2} \\
& +2 \cdot\left(\frac{\partial \theta}{\partial \delta^{25 / 24} M g(A)_{B}} \cdot \frac{\partial \theta}{\partial \delta^{26 / 24} M g(A)_{B}} \cdot u\left(\delta^{25 / 24} M g(A)_{B}\right) \cdot u\left(\delta^{26 / 24} M g(A)_{B}\right) \cdot r\right)
\end{aligned}
$$

For $\theta$-values that are based on isotope ratio measurements by sample-sample bracketing (sample A against sample $\mathrm{B}$ ), the partial derivatives used for the calculation of the uncertainty on $\theta$ are:

$$
\begin{gathered}
\frac{\partial \theta}{\partial \delta^{25 / 24} M g(A)_{B}}=\frac{1}{\left(\ln \left(\left(\delta^{26 / 24} M g(A)_{B}+1000\right) / 1000\right)\right)} \cdot \frac{1}{\left(\delta^{25 / 24} M g(A)_{B}+1000\right)} \\
\frac{\partial \theta}{\partial \delta^{26 / 24} M g(A)_{B}}=-\frac{\ln \left(\left(\delta^{25 / 24} M g(A)_{B}+1000\right) / 1000\right)}{\ln \left(\left(\delta^{26 / 24} M g(A)_{B}+1000\right) / 1000\right)^{2}} \cdot \frac{1}{\left(\delta^{26 / 24} M g(A)_{B}+1000\right)}
\end{gathered}
$$

Supporting equations S-1 and S-6 contain covariance-terms that comprise the uncertainties on $\delta^{25 / 24} \mathrm{Mg}$ and $\delta^{26 / 24} \mathrm{Mg}$ and the empirical correlation coefficient $\mathrm{r}$ that we estimated from three measurements of JDo-1 as 0.94. 


\section{The effect of isotopic difference on the precision of $S$-values}

The standard deviation on apparent $\theta$-values and $S$-values depends to a large degree on the isotopic difference between the samples $\mathrm{A}$ and $\mathrm{B} \Delta^{\mathrm{z} / \mathrm{x}} \mathrm{A}-\mathrm{B}$. This effect we illustrate by comparing the calculated uncertainties on apparent $\theta$-values (for sample-sample bracketing, Supporting equation S6) with standard deviations on empirical $S$-values (i.e. the slope between a bracketing standard and a sample)) that we compiled from published $\delta$-values for $\mathrm{Mg}$ and $\mathrm{Si}$. The standard deviation of $S$-values for a given isotopic difference to $\delta$-zero (i.e., the $\delta$-value) approximates the standard deviation of apparent $\theta$-values measured by sample-sample bracketing.

The match between calculated uncertainties on apparent $\theta$-values and the empirically determined range of $S$-values shows that the measured standard deviation on $S$-values matches the actual uncertainty. Notably, samples with different fractionation histories relative to the standard will have meaningless slopes that can be outside the range of canonical $\theta$-values, an example being the slope $S_{\text {JLs-1 - ERM-AE144 }}$ of $0.493 \pm 0.01(1 \mathrm{SD}, \mathrm{n}=5)$ (Supporting Figure 1A). However, also note that true educt-product pairs can have $\theta$-values outside this range (Bao et al., 2015; c.f. main text).
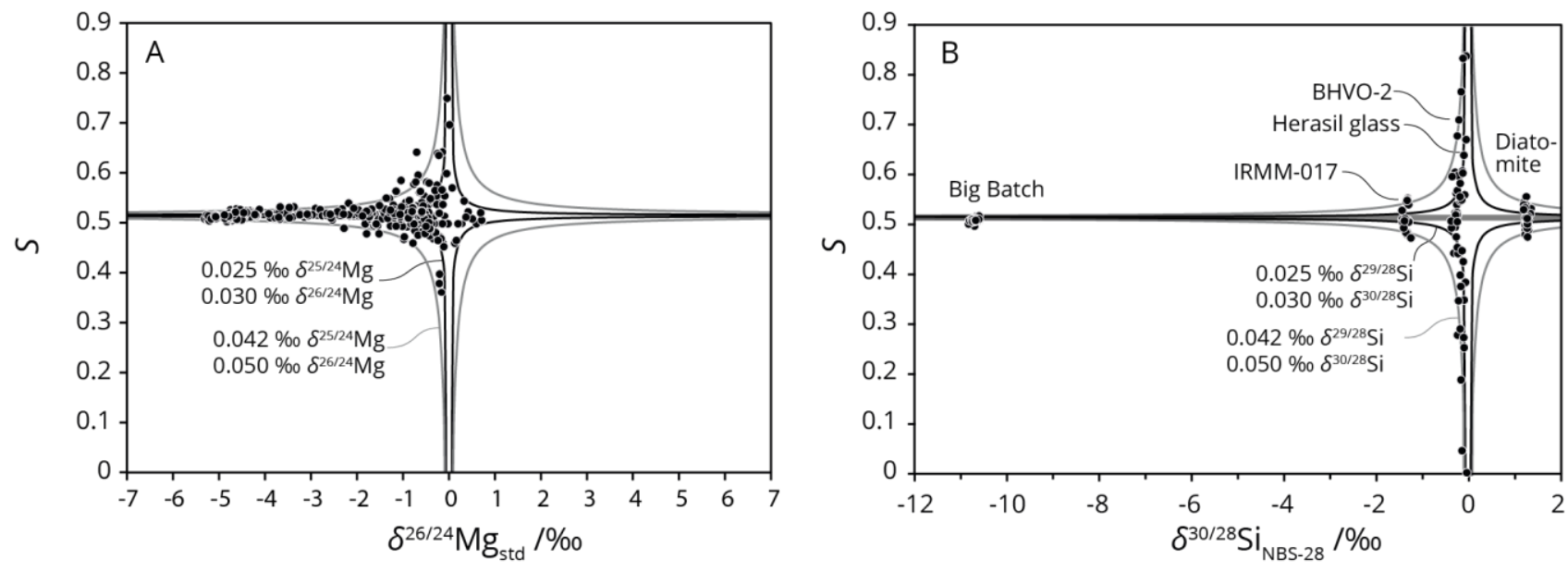

Supporting Figure S1: The $S_{\text {spl-std }}-\delta$ relation using the examples of Mg and Si stable isotopes. The standard deviation of $S$-values between sample and standard decreases with increasing distance from $\delta$-zero, because the relative precision on $S$ increases with increasing isotopic difference. The lines delineate the range of slopes calculated from uncertainties on apparent $\theta$ (main text equation 12) for standard uncertainties on $\delta^{25 / 24} \mathrm{Mg}$ and $\delta^{26 / 24} \mathrm{Mg}$ of $0.040 \%$ and $0.050 \%$ (gray curves) and $0.026 \%$ and $0.033 \%$ (black curves). A) The Mg isotope data is compiled (black dots) from Pogge von Strandmann ${ }^{4}$, Hippler et al. ${ }^{5}$, Immenhauser et al. ${ }^{6}$, Bolou-Bi et $a l .{ }^{7}$, Mavromatis et al. ${ }^{8}$, Martin et al..$^{9,10}$, Geske et al. ${ }^{11,12}$, Pokharel et al. ${ }^{13}$, Uhlig et al. ${ }^{14}$ and is referenced to DSM3. B) The Si isotope data is compiled from Tatzel et al. ${ }^{15,16}$, showing that the standard deviation similarly decreases with isotopic difference for a different isotope system. The curves are identical to the uncertainty curves calculated for $\mathrm{Mg}$. 


\section{Supporting Figure S2}

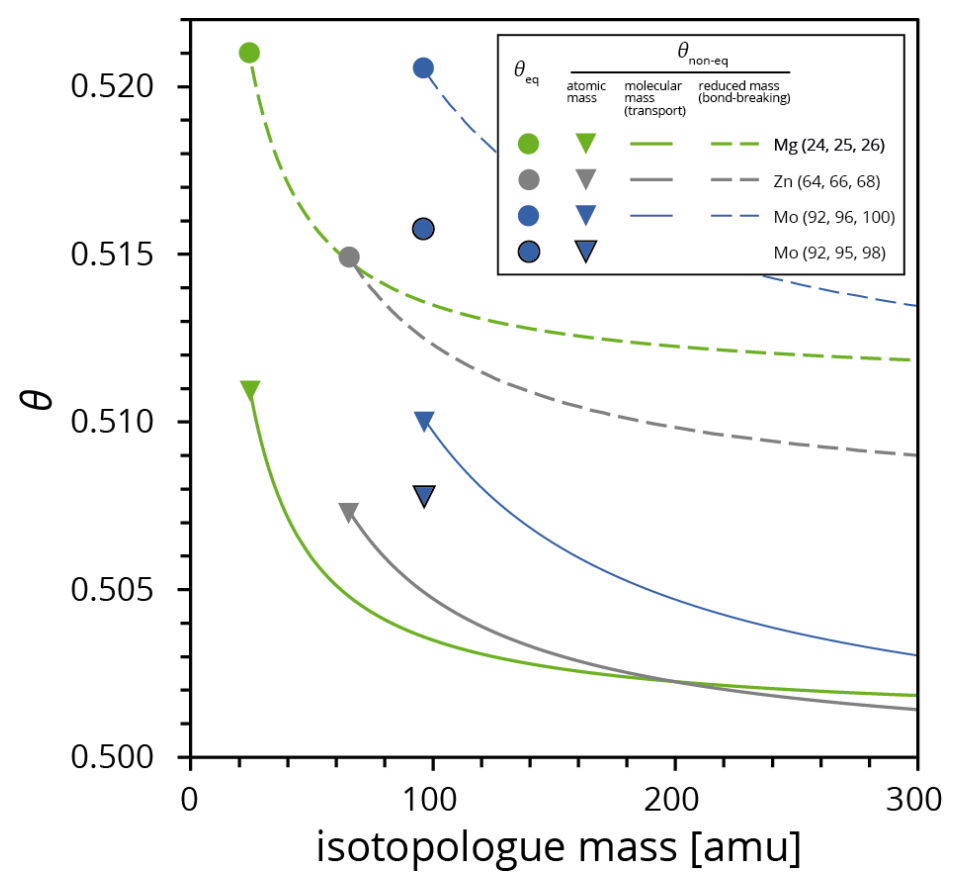

Supporting Figure S2: Theoretical $\theta$-values for different mass-dependent isotope fractionation processes for the examples $\mathbf{M g}, \mathbf{Z n}$ and Mo. $\theta_{\text {eq }}$ is a high-temperature equilibrium end-member (eqn. 15 in Young et $\mathrm{al}^{17}$ ). Non-equilibrium effects for transport and bond-breaking of isotopologues are calculated from eqn. 21 in Young et $\mathrm{al}^{17}$ ). In some cases (example Mo), the resolution of $\theta_{\text {app }}$-values can be increased when isotopes are selected accordingly (see main text).

\section{Supporting Figure S3}
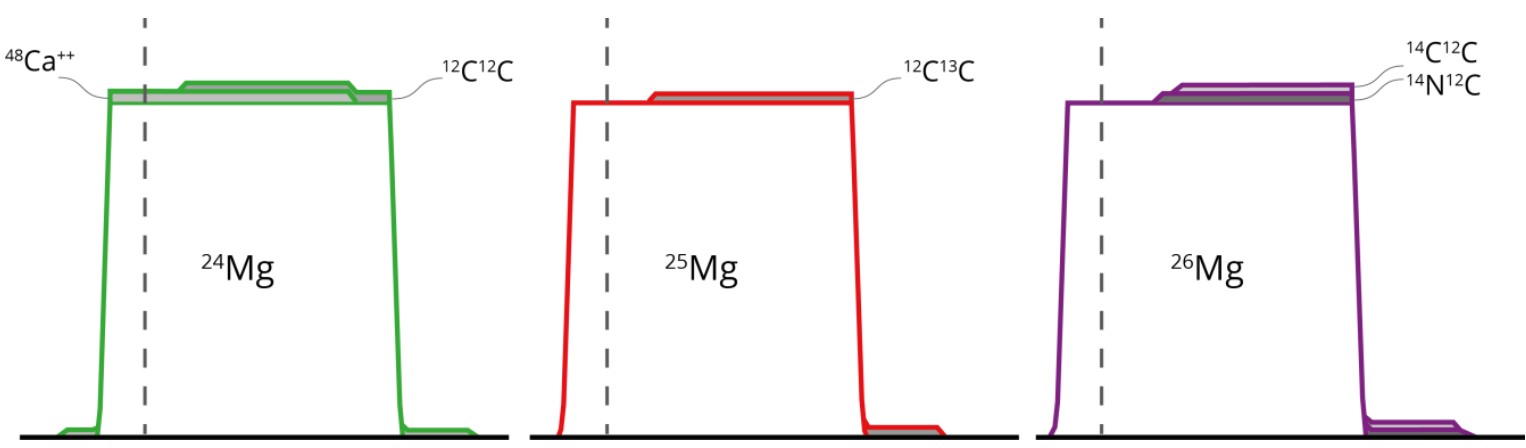

Supporting Figure S3: Schematic mass-spectra of $\mathrm{Mg}$ showing the predominant interferences. Interference-free measurement of $\mathrm{Mg}$ isotope ratios requires quantitative separation of $\mathrm{Ca}$ and measurement on the low-mass side of the mass spectrum (dashed line). 


\section{Supporting Figure S4}

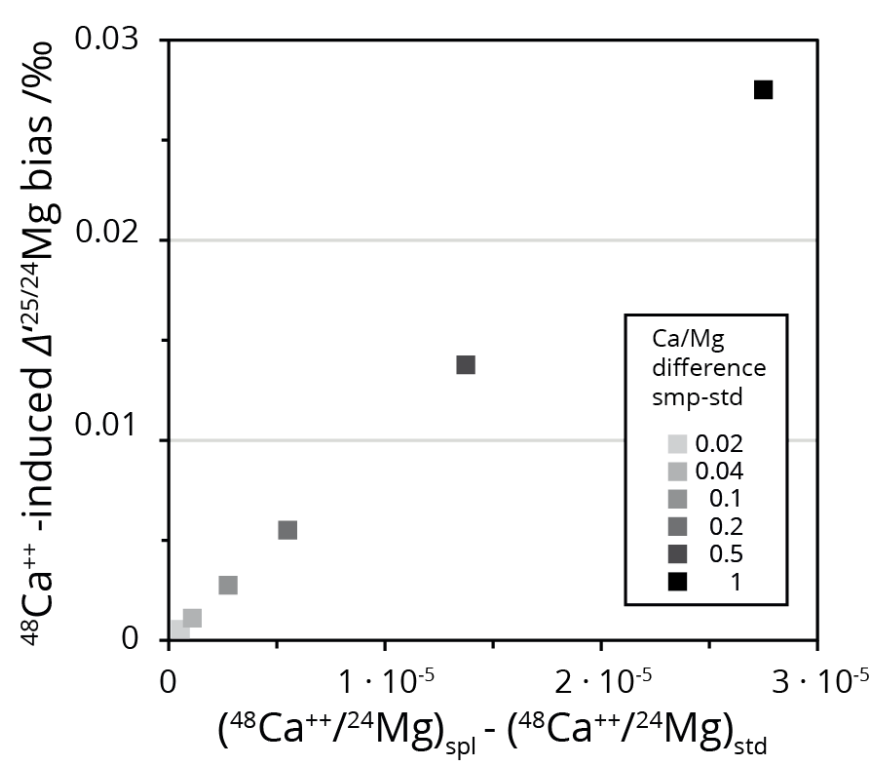

Supporting Figure S4: Bias in $\Delta^{625 / 24} \mathrm{Mg}$ resulting from the ${ }^{48} \mathrm{Ca}^{++}$interference on ${ }^{24} \mathrm{Mg}$. The effect is calculated based on measured ${ }^{48} \mathrm{Ca}^{++}$count rates of $1.8 \mathrm{cts} / \mathrm{ppb} \mathrm{Ca}$. 


\section{Supporting Table S1}

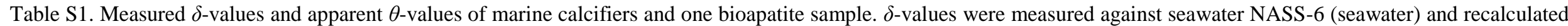
against DSM3 using supporting equation S9.

\begin{tabular}{|c|c|c|c|c|c|c|c|c|c|c|}
\hline sample & species & $\begin{array}{c}\delta^{25 / 24} \mathrm{Mg}_{\text {NASS-6 }} \\
(\% \mathrm{o})\end{array}$ & $2 \mathrm{SD}$ & $\begin{array}{c}\delta^{26 / 24} \mathrm{Mg}_{\text {NASS-6 }} \\
(\% 0)\end{array}$ & $2 \mathrm{SD}$ & $\theta_{\text {app }}$ & $2 S D$ & $\begin{array}{c}\delta^{25 / 24} \mathrm{Mg}_{\mathrm{DSM} 3} \\
(\% \mathrm{o})\end{array}$ & $\begin{array}{c}\delta^{26 / 24} \mathrm{Mg}_{\mathrm{DSM} 3} \\
(\% \mathrm{\%})\end{array}$ & $\mathrm{n}$ \\
\hline MC120 & Globorotalia menardii & -1.98 & 0.012 & -3.84 & 0.012 & 0.514 & 0.005 & -2.39 & -4.66 & 6 \\
\hline JCp-1 & Porites (JCp-1) & -0.61 & 0.005 & -1.19 & 0.007 & 0.515 & 0.006 & -1.03 & -2.00 & 6 \\
\hline EN-1 & Tridacna (EN-1) & -1.82 & 0.016 & -3.57 & 0.022 & 0.508 & 0.007 & -2.23 & -4.39 & 7 \\
\hline JCt-1 & Tridacna (JCt-1) & -1.30 & 0.009 & -2.55 & 0.007 & 0.509 & 0.006 & -1.72 & -3.37 & 7 \\
\hline D Hai6 & Carcharodon carcharias & -0.25 & 0.013 & -0.48 & 0.010 & 0.511 & 0.040 & -0.66 & -1.30 & 7 \\
\hline
\end{tabular}

\section{Supporting Table S2}

Supporting Table S2. Measured $\delta$-values of reference materials. The measurements were done by standard-sample bracketing against ERM-AE144 and recalculated versus DSM3 using supporting equation 10 . Reference values of JDo- 1 are $\delta^{25 / 24} \mathrm{Mg}_{\mathrm{DSM} 3}=-1.23 \%$, $\delta^{26 / 24} \mathrm{Mg}_{\mathrm{DSM} 3}=-2.32 \% \mathrm{o}^{1}$, and of $\mathrm{BHVO}-2 \delta^{25 / 24} \mathrm{Mg}_{\mathrm{DSM} 3}=-0.12 \%$, $\delta^{26 / 24} \mathrm{Mg}_{\mathrm{DSM} 3}=-0.24 \%{ }^{18}$.

\begin{tabular}{|c|c|c|c|c|c|c|c|c|}
\hline sample & $\begin{array}{c}\delta^{25 / 24} \mathrm{Mg}_{\text {ERM-AE144 }} \\
(\% \text { o })\end{array}$ & 2 SD & $\begin{array}{c}\delta^{26 / 24} \mathrm{Mg}_{\text {ERM-AE } 144} \\
(\% 0)\end{array}$ & 2 SD & $\begin{array}{c}\delta^{25 / 24} \mathrm{Mg}_{\mathrm{DSM} 3} \\
(\% \mathrm{o})\end{array}$ & $\begin{array}{c}\delta^{26 / 24} \mathrm{MgDSM}_{\mathrm{DS}} \\
(\% \mathrm{o})\end{array}$ & $n$ & material \\
\hline BHVO-2 & 2.38 & 0.004 & 4.63 & 0.04 & -0.14 & -0.29 & 3 & basalt \\
\hline JDo-1 & 1.32 & 0.04 & 2.61 & 0.05 & -1.20 & -2.32 & 14 & dolomite \\
\hline JLs-1-2 & 0.66 & 0.05 & 1.34 & 0.07 & -1.86 & -3.60 & 3 & calcite \\
\hline NASS-6 & 2.09 & 0.03 & 4.10 & 0.05 & -0.43 & -0.83 & 19 & seawater \\
\hline
\end{tabular}




\section{$\underline{\text { Supporting Table S3 }}$}

Supporting Table S3: Measured $\delta$-values of standards analyzed by standard-sample bracketing against DSM3 and ERM-AE144, respectively.

\begin{tabular}{lcc|cc|} 
& $\begin{array}{c}\delta^{25 / 24} \begin{array}{c}\text { Mgdsm3 } \\
(\%)\end{array} \\
\text { ERM-AE143 }\end{array}$ & $1 \mathrm{SD}$ & $\begin{array}{c}\delta^{26 / 24} \mathbf{M g}_{\text {DSM3 }} \\
(\% \mathrm{o})\end{array}$ & $1 \mathrm{SD}$ \\
ERM-AE144 & -1.72 & 0.01 & -3.35 & 0.03 \\
ERM-AE145 & -2.53 & 0.02 & -4.95 & 0.04 \\
\hline
\end{tabular}

\begin{tabular}{|c|c|c|c|c|c|c|}
\hline & & & & & Reference values & Vogl et al., 2016) \\
\hline & $\begin{array}{c}\delta^{25 / 24} \mathrm{Mg}_{\text {ERM-AE } 144} \\
(\% \circ)\end{array}$ & $1 \mathrm{SD}$ & $\begin{array}{c}\delta^{26 / 24} \mathrm{Mg}_{\text {ERM-AE } 144} \\
(\%)\end{array}$ & $1 \mathrm{SD}$ & $\begin{array}{c}\delta^{25 / 24} \mathrm{Mg}_{\text {ERM-AE } 144} \\
(\% 0)\end{array}$ & $\begin{array}{c}\delta^{26 / 24} \mathrm{Mg}_{\text {ERM-AE } 144} \\
(\% \circ)\end{array}$ \\
\hline ERM-AE143 & 0.82 & 0.03 & 1.63 & 0.04 & 0.82 & 1.61 \\
\hline ERM-AE145 & 0.21 & 0.02 & 0.32 & 0.02 & 0.22 & 0.34 \\
\hline
\end{tabular}




\section{Supporting Table S4}

Supporting Table S4. Linearized $\delta$-values of separate splits of the Mg fraction eluted from DOWEX W50-X12 cation exchange resin (main text Fig. 8).

\begin{tabular}{lccccc} 
& $\begin{array}{c}\delta^{25 / 24} \mathrm{Mg}_{\text {DSM3 }} \\
(\% \circ)\end{array}$ & $2 \mathrm{SD}$ & $\begin{array}{c}\delta^{26 / 24} \mathrm{Mg}_{\mathrm{DSM} 3} \\
(\% \circ)\end{array}$ & $2 \mathrm{SD}$ & $\mathrm{n}$ \\
\hline $\mathrm{mL} \mathrm{1-2}$ & -1.14 & 0.03 & -2.24 & 0.04 & 6 \\
$\mathrm{~mL} \mathrm{3}$ & -1.86 & 0.02 & -3.64 & 0.04 & 6 \\
$\mathrm{~mL} \mathrm{4-5}$ & -2.82 & 0.04 & -5.47 & 0.03 & 6 \\
$\mathrm{~mL} \mathrm{6-8}$ & -4.15 & 0.05 & -8.03 & 0.07 & 4 \\
educt & -1.95 & 0.02 & -3.80 & 0.06 & 6
\end{tabular}

\section{Supporting Equation S9:}

$\delta$-values can be converted into another $\delta$-value reference frame using equation S9 ${ }^{19}$ for $\delta$-values obtained from eqn. 1 (main article) in their basic form (no \%, ppm, etc.). When $\delta$-values in \%o are used, they have to be divided by 1000 before entering into equation S9.

$$
\begin{aligned}
\delta^{\mathrm{y}, \mathrm{z} / \mathrm{x}} E(s p l)_{s t d B} & =\delta^{\mathrm{y}, \mathrm{z} / \mathrm{x}} E(s p l)_{s t d A}-\delta^{\mathrm{y}, \mathrm{z} / \mathrm{x}} E(\operatorname{std} B)_{s t d A} \\
& +\left(\delta^{\mathrm{y}, \mathrm{z} / \mathrm{x}} E(s p l)_{s t d A} \cdot \delta^{\mathrm{y}, \mathrm{z} / \mathrm{x}} E(\operatorname{std} B)_{s t d A}\right)
\end{aligned}
$$

\section{Supporting References}

(1) Shalev, N.; Farkas, J.; Fietzke, J.; Novak, M.; Schuessler, J. A.; Pogge von Strandmann, P. A. E.; Törber, P. B. Mg Isotope Inter-Laboratory Comparison of Reference Materials from EarthSurface Low-Temperature Environments. Geostand. Geoanalytical Res. 2018, 42, 205-221. https://doi.org/10.1111/ggr.12208.

(2) Vogl, J.; Brandt, B.; Rienitz, O.; Noordmann, J.; Malinovskiy, D. Certification Report for the Isotope Reference Materials ERM®-AE143, ERM®-AE144 and ERM®-AE145; 2018.

(3) Bao, H.; Cao, X.; Hayles, J. A. The Confines of Triple Oxygen Isotope Exponents in Elemental and Complex Mass-Dependent Processes. Geochim. Cosmochim. Acta 2015, 170, 39-50. https://doi.org/10.1016/j.gca.2015.07.038.

(4) Pogge von Strandmann, P. A. E. Precise Magnesium Isotope Measurements in Core Top Planktic and Benthic Foraminifera. Geochemistry, Geophys. Geosystems 2008, 9 (12). https://doi.org/10.1029/2008GC002209.

(5) Hippler, D.; Buhl, D.; Witbaard, R.; Richter, D. K.; Immenhauser, A. Towards a Better Understanding of Magnesium-Isotope Ratios from Marine Skeletal Carbonates. Geochim. Cosmochim. Acta 2009, 73 (20), 6134-6146. https://doi.org/10.1016/j.gca.2009.07.031.

(6) Immenhauser, A.; Buhl, D.; Richter, D.; Niedermayr, A.; Riechelmann, D.; Dietzel, M.; Schulte, U. Magnesium-Isotope Fractionation during Low-Mg Calcite Precipitation in a Limestone Cave - Field Study and Experiments. Geochim. Cosmochim. Acta 2010, 74 (15), 4346-4364. https://doi.org/10.1016/j.gca.2010.05.006. 
(7) Bolou-Bi, E. B.; Vigier, N.; Poszwa, A.; Boudot, J.-P. P.; Dambrine, E. Effects of Biogeochemical Processes on Magnesium Isotope Variations in a Forested Catchment in the Vosges Mountains (France). Geochim. Cosmochim. Acta 2012, 87, 341-355. https://doi.org/10.1016/j.gca.2012.04.005.

(8) Mavromatis, V.; Pearce, C. R.; Shirokova, L. S.; Bundeleva, I. A.; Pokrovsky, O. S.; Benezeth, P.; Oelkers, E. H. Magnesium Isotope Fractionation during Hydrous Magnesium Carbonate Precipitation with and without Cyanobacteria. Geochim. Cosmochim. Acta 2012, 76, 161-174. https://doi.org/10.1016/j.gca.2011.10.019.

(9) Martin, J. E.; Vance, D.; Balter, V. Natural Variation of Magnesium Isotopes in Mammal Bones and Teeth from Two South African Trophic Chains. Geochim. Cosmochim. Acta 2014, 130, 12-20. https://doi.org/10.1016/j.gca.2013.12.029.

(10) Martin, J. E.; Vance, D.; Balter, V. Magnesium Stable Isotope Ecology Using Mammal Tooth Enamel. Proc. Natl. Acad. Sci. 2015, $112 \quad$ (2), 430-435. https://doi.org/10.1073/pnas.1417792112.

(11) Geske, A.; Lokier, S.; Dietzel, M.; Richter, D. K.; Buhl, D.; Immenhauser, A. Magnesium Isotope Composition of Sabkha Porewater and Related (Sub-)Recent Stoichiometric Dolomites, Abu Dhabi (UAE). Chem. Geol. 2015, 393-394, 112-124. https://doi.org/10.1016/j.chemgeo.2014.11.020.

(12) Geske, A.; Goldstein, R. H.; Mavromatis, V.; Richter, D. K.; Buhl, D.; Kluge, T.; John, C. M.; Immenhauser, A. The Magnesium Isotope $(\Delta 26 \mathrm{Mg})$ Signature of Dolomites. Geochim. Cosmochim. Acta 2015, 149, 131-151. https://doi.org/10.1016/j.gca.2014.11.003.

(13) Pokharel, R.; Gerrits, R.; Schuessler, J. A.; Floor, G. H.; Gorbushina, A. A.; von Blanckenburg, F. Mg Isotope Fractionation during Uptake by a Rock-Inhabiting, Model Microcolonial Fungus Knufia Petricola at Acidic and Neutral PH. Environ. Sci. Technol. 2017, 51 (17), 9691-9699. https://doi.org/10.1021/acs.est.7b01798.

(14) Uhlig, D.; Schuessler, J. A.; Bouchez, J.; Dixon, J. L.; von Blanckenburg, F. Quantifying Nutrient Uptake as Driver of Rock Weathering in Forest Ecosystems by Magnesium Stable Isotopes. Biogeosciences 2017, 14 (January), 3111-3128. https://doi.org/10.5194/bg-2016-521.

(15) Tatzel, M.; von Blanckenburg, F.; Oelze, M.; Schuessler, J. A.; Bohrmann, G. The Silicon Isotope Record of Early Silica Diagenesis. Earth Planet. Sci. Lett. 2015, 428, 293-303. https://doi.org/10.1016/j.epsl.2015.07.018.

(16) Tatzel, M.; von Blanckenburg, F.; Oelze, M.; Bouchez, J.; Hippler, D. Late Neoproterozoic Seawater Oxygenation by Siliceous Sponges. Nat. Commun. 2017, 8 (1), 621. https://doi.org/10.1038/s41467-017-00586-5.

(17) Young, E. D.; Galy, A.; Nagahara, H. Kinetic and Equilibrium Mass-Dependant Isotope Fractionation Laws in Nature and Their Geochemical and Cosmochemical Significance. Geochim. Cosmochim. Acta 2002, 66 (6), 1095-1104. https://doi.org/10.1016/S00167037(01)00832-8.

(18) Teng, F.-Z. Magnesium Isotope Geochemistry. Rev. Mineral. Geochemistry 2017, 82 (1), 219287. https://doi.org/10.2138/rmg.2017.82.7.

(19) Vogl, J.; Pritzkow, W. Isotope Reference Materials for Present and Future Isotope Research. J. Anal. At. Spectrom. 2010, 25 (7), 923-932. https://doi.org/10.1039/c000509f. 